\title{
Nonconventional magnetic phenomena in neodymium thin film
}

\author{
G. Yumnam, ${ }^{1}$ J. Guo $\odot,{ }^{1}$ Y. Chen, ${ }^{1}$ V. Lauter, ${ }^{2}$ and D. K. Singh ${ }^{1, *}$ \\ ${ }^{1}$ Department of Physics and Astronomy, University of Missouri, Columbia, Missouri 65211, USA \\ ${ }^{2}$ Neutron Scattering Division, Oak Ridge National Laboratory, Oak Ridge, Tennessee 37830, USA
}

(Received 24 April 2020; accepted 14 September 2020; published 2 October 2020)

\begin{abstract}
Neodymium is a remarkable active component in numerous magnetic alloys that are used in various applications. However, the application of a bare neodymium thin film is limited due to the lack of information about its electrical and magnetic properties. We report a synergistic study of an $\mathrm{Nd}$ thin film using experimental and theoretical techniques of polarized neutron reflectometry, magnetoresistance measurement, and density functional theory. Unlike bulk $\mathrm{Nd}$, the thin film specimen is a very poor electrical conductor. Also, as grown, the thin film on silicon substrate does not exhibit any magnetism in a zero field. However, moderate in-plane field application of $H=1.2 \mathrm{~T}$ tends to induce weak magnetism in the system at a low temperature of $T<18 \mathrm{~K}$, which coincides with an unusual cross-over behavior in magnetoresistance. The study provides important insight in the physical characteristics of the Nd thin film that are atypical for a magnetic system.
\end{abstract}

DOI: 10.1103/PhysRevResearch.2.043018

Neodymium magnet's prosaic use includes a long list of applications [1]. Some of the examples include uses in the alloys to make a magnet for household purposes, hard disk drive for data storage, magnetic resonance imaging machine, and colored glasses and light bulbs [2-7]. A majority of the applications hinge on understanding the physical and magnetic characteristics of neodymium (Nd) in bulk and thin film specimens [8,9]. Besides the practical applications, the $\mathrm{Nd}$ element is an integral component in the numerous scientific investigations of fundamental importance in solid state and materials physics. For instance, Nd-based compounds are at the forefront of the study of quantum magnetism or the exploration of plasmonic properties [10-15]. More recently, an emergent nanomaterial with a honeycomb motif, created using the Nd element, was used to demonstrate a highly novel state of the Wigner crystal of magnetic charges [16]. In the bulk form, neodymium is argued to manifest antiferromagnetic spin-density-wave-type order below $T_{N} \simeq 19 \mathrm{~K}[17,18]$. However, little or no information is available about the neodymium thin film; especially electrical and magnetic properties that form the core of physical understanding, necessary for the practical applications. Here, we report on a comprehensive study of neodymium thin film using the synergistic experimental and theoretical investigations. Experimental research, performed using electrical, magnetoresistance, and polarized neutron reflectometry measurements, are complemented by the first-principle theoretical calculations under the ambit of the density functional theory formalism. Unlike bulk Nd with

\footnotetext{
*singhdk@missouri.edu

Published by the American Physical Society under the terms of the Creative Commons Attribution 4.0 International license. Further distribution of this work must maintain attribution to the author(s) and the published article's title, journal citation, and DOI.
}

metallic characteristic $[19,20]$, the thin film of $\mathrm{Nd}$ is found to exhibit very poor electrical conduction property. Interestingly, a cross-over behavior between negative and positive magnetoresistance is found to occur at $T \simeq 18 \mathrm{~K}$. An in-plane magnetic-field application tends to induce a weak magnetic order at low temperature, which is otherwise absent in the thin film sample.

Polycrystalline thin film of neodymium, thickness $\simeq 20 \mathrm{~nm}$, is created using electron-beam deposition of neodymium vapor on the silicon substrate in an ultra-high vacuum chamber. For e-beam deposition, the high purity ( $99.9 \%$ or higher) Nd pallets were commercially obtained from Kurt J. Lesker. The four probe technique was employed to measure the electrical resistance and magnetoresistance of the $\mathrm{Nd}$ thin film. Electrical measurements were performed in a closed-cycle refrigerator-cooled $9 T$ magnet with the measurement temperature range of $T=5 \mathrm{~K}$ to $300 \mathrm{~K}$. Due to the high electrical resistance, electrical measurements were carried out using the synchronized combination of Keithley 6221 current source meter and 2182 nanovoltmeter in the delta mode configuration. Polarized neutron reflectometry (PNR) measurements were performed on a $20 \times 20 \mathrm{~mm}^{2}$ surface area sample at the Magnetism Reflectometer at beam line BL-4A of the Spallation Neutron Source (SNS) at the Oak Ridge National Laboratory [21]. The instrument operates using the time-offlight technique in a horizontal scattering geometry with a bandwidth of 5.6 $\AA$ (wavelength varying between 2.6-8.2 $\AA$ ). The beam was collimated using a set of slits before the sample and measured with a two-dimensional position sensitive ${ }^{3} \mathrm{He}$ detector with $1.5-\mathrm{mm}$ resolution at $2.5 \mathrm{~m}$ from the sample. The sample was mounted on the copper cold finger of a close cycle refrigerator with a base temperature of $T=$ $5 \mathrm{~K}$. Beam polarization was performed using the reflective supermirror devices, achieving better than $98 \%$ polarization efficiency over the full wavelength band. Also, the full vertical divergence was used for maximum intensity and a $5 \%$ 

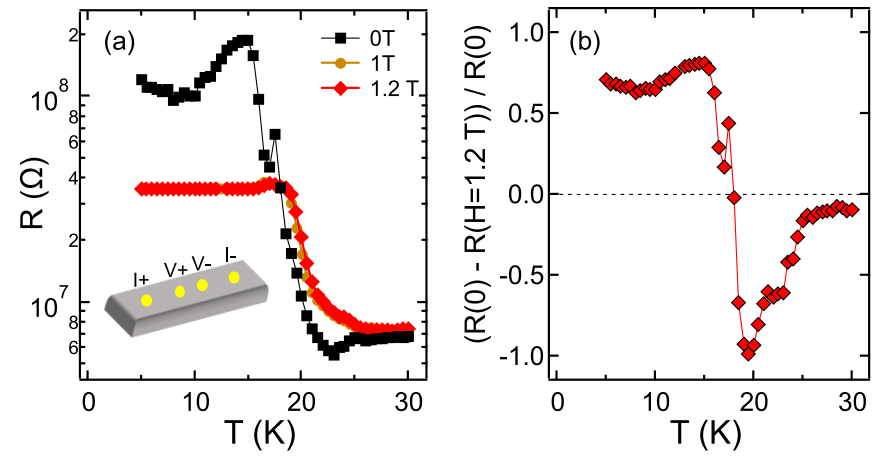

FIG. 1. Electrical characterization of Nd thin film. (a) Electrical resistance as a function of temperature at $H=0 \mathrm{~T}$ and $H=1.2 \mathrm{~T}$. A clear separation between the two resistance curves occur at $T \simeq$ $25 \mathrm{~K}$. Below $T \simeq 18 \mathrm{~K}$, zero field resistance exceeds the field data. Inset shows the four-probe measurement scheme. (b) MR vs. temperature. Remarkable crossover from negative to positive MR occurs around $T \simeq 18 \mathrm{~K}$.

angular relative resolution $\Delta \theta / \theta \simeq \Delta q_{z} / q_{z}$ in the horizontal direction. Measurement in the magnetic field utilized an electromagnet with a maximum field of $H=1.2 \mathrm{~T}$, applied in-plane to the sample.

We plot the electrical resistance of the $\mathrm{Nd}$ film as a function of temperature at $H=0 \mathrm{~T}$ and $H=1.2 \mathrm{~T}$ field in Fig. 1(a). The highly resistive nature of the film becomes immediately noticeable. As temperature reduces, a steep rise in resistance is detected below $T \simeq 25 \mathrm{~K}$ in the $H=0 \mathrm{~T}$ field data. However, the resistivity tends to flatten for further decrease in temperature below $T \simeq 15 \mathrm{~K}$ (albeit, preceded by a spike). Although, the sharp upward trend in electrical resistance is usually ascribed to the thermally activated behavior, the flattening characteristic at further lower temperature suggests a competing intrinsic mechanism at play. This argument is further substantiated by comparing the $H=0 \mathrm{~T}$ measurement to the $H=1.2 \mathrm{~T}$ data in Fig. 1(a). Three important observations can be inferred here: (a) the resistivity curve at $H=1.2 \mathrm{~T}$ separates out from the $H=0$ curve below $\mathrm{T}=$ $25 \mathrm{~K}$, suggesting the onset of magnetism below $T \simeq 25 \mathrm{~K}$ in the Nd thin film; (b) resistivity curves tend to flatten below $T \simeq 20 \mathrm{~K}(H=1.2 \mathrm{~T})$ or $T \simeq 15 \mathrm{~K}(H=0 \mathrm{~T})$, and (c) $R(H)$ is significantly smaller than $R(0)$ below $T \simeq 18 \mathrm{~K}$. The reduction in electrical resistance below $T \simeq 18 \mathrm{~K}$ in an applied field is most likely arising due to the field-induced alignment of the magnetic moment along the field direction; thus, causing a ferromagnetic-type moment arrangement in the system. Ferromagnetic configuration is known to give rise to lower resistance in magnetic materials $[22,23]$. We further understand it by plotting magnetoresistance (MR), given by $[R(0)-R(H=1.2 T)] / R(0)$, as a function of temperature in Fig. 1(b). The plot of MR versus temperature exhibits a very interesting cross-over behavior from -ve values, at $18 \mathrm{~K}$ $<T<25 \mathrm{~K}$, to $+v e$ values at $T<18 \mathrm{~K}$. Negative magnetoresistance is often associated to the variable range hopping mechanism of charge carriers between the exchange coupled magnetic clusters in a magnetic material $[24,25]$. Therefore, electrical conduction, in the temperature range of $18 \mathrm{~K}<T<$ $25 \mathrm{~K}$, may be occurring due to the hopping of charge carriers.
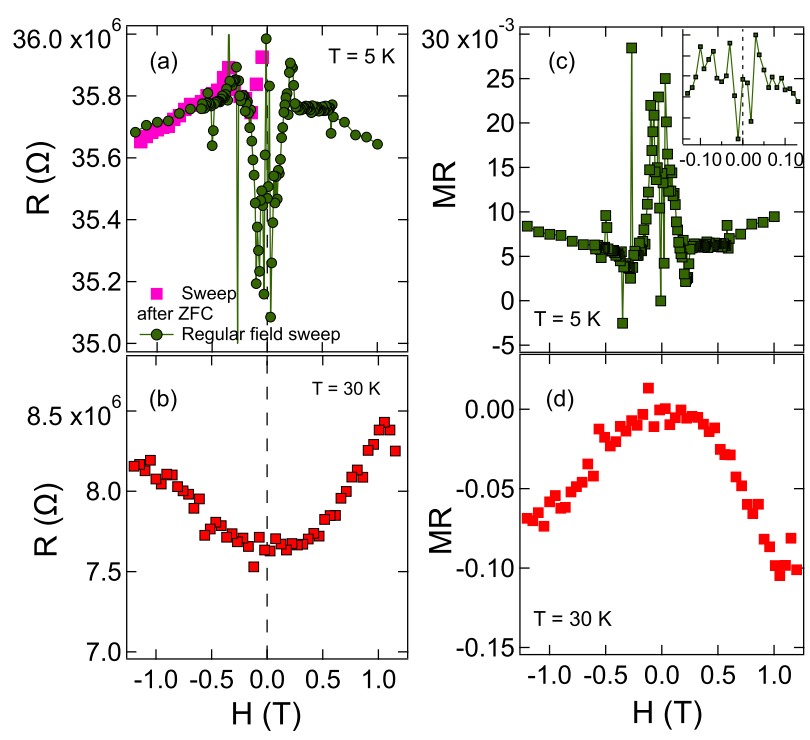

FIG. 2. Magnetoresistance measurement of $\mathrm{Nd}$ film. (a), (b) $R$ vs. $H$ at $T=5 \mathrm{~K}$ and $T=30 \mathrm{~K}$. Unlike the resistance vs. field at $T=30 \mathrm{~K}$, complex behavior is observed near zero field in $T=5 \mathrm{~K}$ data. Pink color marker is from the zero-field cooled measurement at $T=5 \mathrm{~K}$, immediately after cooling the sample from $T=300 \mathrm{~K}$ to $T=5 \mathrm{~K}$ in zero magnetic field. (c), (d) $\mathrm{MR}$, given by $[\mathrm{R}(0)$ $\mathrm{R}(H)] / \mathrm{R}(0)$, vs. $H$ at $T=5 \mathrm{~K}$ and $T=30 \mathrm{~K}$. Resistance in zero-field cool data at $H=0 \mathrm{~T}$ is used as $R(0)$, which is higher than the value in field sweep mode. MR data at different temperatures exhibit opposite trends in field, e.g., MR at $T=30 \mathrm{~K}$ exhibits linear decrement, typically observed in a paramagnetic system, as magnetic field increases, while the MR at $T=5 \mathrm{~K}$ increases as a function of field at $H>400 \mathrm{Oe}$. At low field, highly complex trends in MR, indicating the existence of quasistable states at $T=5 \mathrm{~K}$, are detected (see inset in c).

On the other hand, the positive value of MR is argued to be arising due to the thermally activated mechanism in magnetic systems [26]. However, this argument is not applicable to electrical resistance temperature dependence below $T \simeq 18 \mathrm{~K}$ in the Nd thin film, as it does not manifest exponential rise as a function of (reducing) temperature. Rather resistance flattens at lower temperature. At $T<18 \mathrm{~K}$, the system tends to develops weak field-induced magnetism.

The quantitative information about field-induced magnetism in the Nd thin film can be obtained from analyzing the magnetoresistance data as a function of applied magnetic field. MR data as a function of magnetic field were acquired at two temperatures of $T=5 \mathrm{~K}$ (in the field-induced magnetism regime) and $T=30 \mathrm{~K}$ (above the magnetism onset temperature). For MR measurement purposes, the sample was cooled from $T=300 \mathrm{~K}$ to $T=5 \mathrm{~K}$ in zero field. Plots of magnetoresistance as a function of magnetic field in the Nd thin film are shown in Fig. 2. Distinct electrical responses to applied field are observed in $T=5 \mathrm{~K}$ and $T=$ $30 \mathrm{~K}$ data. While a uniform linear increment is observed in negative MR as a function of magnetic field at $T=30 \mathrm{~K}$, measurements at $T=5 \mathrm{~K}$ reveal complex behavior. The linear variation in negative MR at $T=30 \mathrm{~K}$ is a typical characteristic of paramagnetic system [27]. The thin film behaves as a 

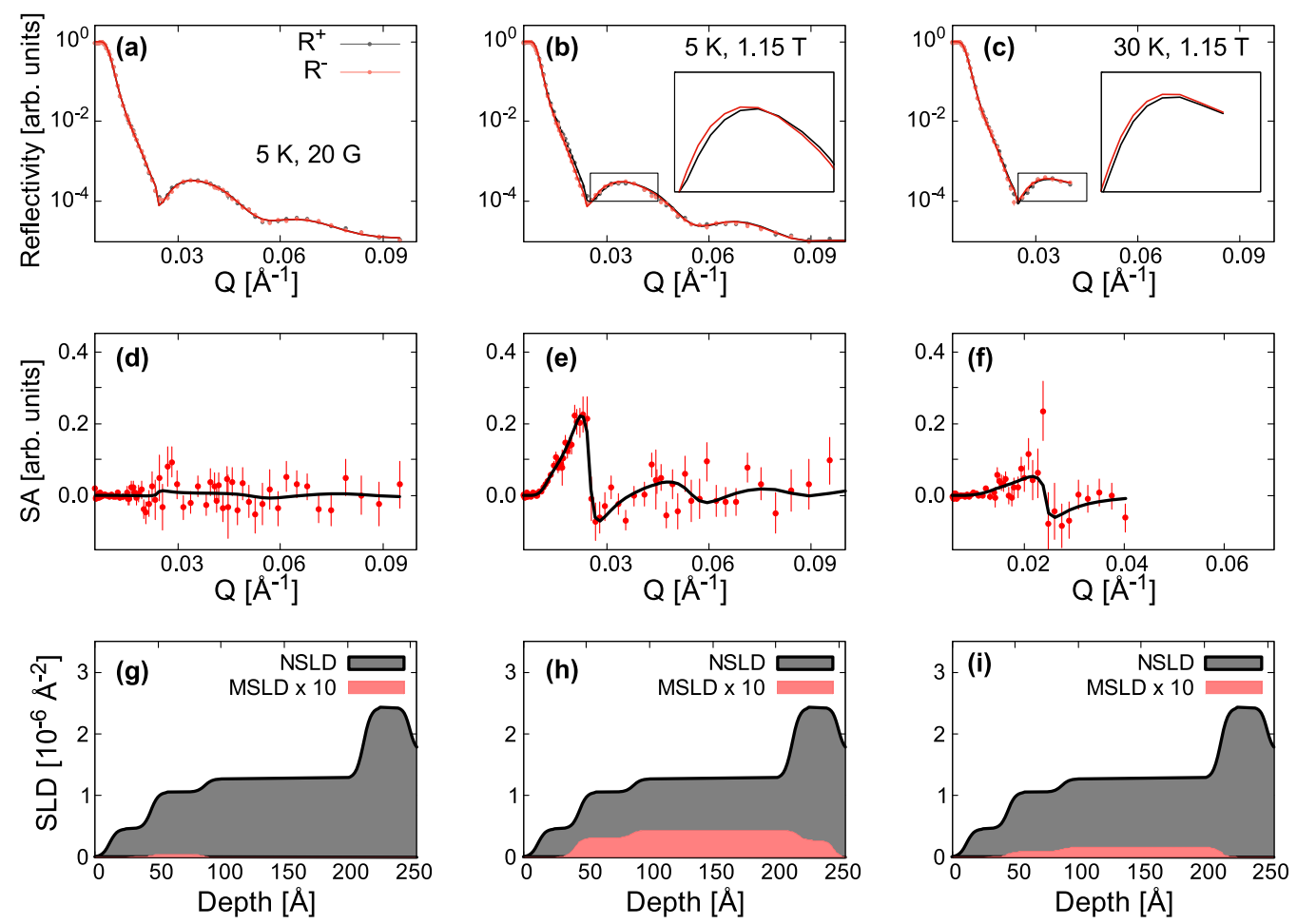

FIG. 3. Polarized neutron reflectometry measurements on Nd thin film. (a)-(c) A small guide field of $H=20$ Oe is applied to keep neutron polarized. Measured and fitted (solid curves) reflectivity curves for neutron with spin-up $\left(R^{+}\right)$and spin-down $\left(R^{-}\right)$polarizations as a function of wave vector transfer at different temperatures and magnetic fields. (d)-(f) Nuclear and magnetic scattering length density profiles, obtained from the fit to experimental data, as a function of depth of $\mathrm{Nd}$ thin film. (g)-(i) Plot of spin asymmetry SA at different temperatures and fields, obtained from the experimental and fitted reflectivities in (a)-(c). A very small MSLD is extracted in the data at $T=5 \mathrm{~K}$ at $H=1.2 \mathrm{~T}$. No magnetism is inferred to arise in zero magnetic field.

paramagnet at higher temperature of $T>25 \mathrm{~K}$. An entirely different picture emerges in the MR measurement at low temperature. In an unusual observation, MR first decreases to a near zero value, $\simeq 0.002$, at low field of $H=150$ Oe, before quickly bouncing back by an order of magnitude to the highest MR of 0.023 at $H=300$ Oe [see inset in Fig. 2(c)]. It seems that the system undergoes quasistable states near zero field, because the zero field cool magnetoresistance measurement, performed immediately after the sample was cooled from $T=300 \mathrm{~K}$ to $5 \mathrm{~K}$, also exhibits a small dip in resistivity near the zero field. When the magnetic field is swept from the negative value to the positive value (and vice versa), a large change in MR is observed near the zero field. It could be arising due to a competing mechanism where the system tries to achieve the zero-field state and thus minimize the energy, but the remnant magnetization in the sample due to magnetic field application inhibits that tendency $[23,28]$. As a result, the $\mathrm{Nd}$ film develops a quasistable configuration near zero field. Following the unusual back and forth bounces between the high and low MR near zero field, sanity returns as the field increases further. The MR exhibits a gradual increment at higher field. However, the overall magnetoresistance is very small, 0.01 at $H=1.2 \mathrm{~T}$, which suggests the existence of weak field-induced magnetism in the system.

A more direct evidence to the field-induced magnetism in the $\mathrm{Nd}$ thin film is obtained from the polarized neutron reflectometry (PNR) measurements [29]. PNR measurements were performed in a small guide field of $H=20$ Oe to maintain the polarization of the incident and scattered neutron. Experimental data were collected at $T=30 \mathrm{~K}$ and $T=5 \mathrm{~K}$ in the zero and high magnetic field of $H=1.2 \mathrm{~T}$, applied in-plane to the sample. We show the plots of specular reflectivities $R^{+}$and $R^{-}$("+" and "-" correspond to a neutron with spin parallel and antiparallel to the guided magnetic field) at $T=5 \mathrm{~K}$ in $H=0 \mathrm{~T}$ and $H=1.2 \mathrm{~T}$ as well as at $T=30 \mathrm{~K}$ in Figs. 3(a) to 3(c). Unlike a ferromagnetic thin film or the nanostructured material where the reflectivities $R^{+}$and $R^{-}$are clearly separated from each other [29,30], indicating the presence of net magnetic moment in the system, the $\mathrm{Nd}$ thin film does not manifest such behavior (beyond two sigma of error bar) in $H=0 \mathrm{~T}$ data at either temperature. It can occur for two reasons: (a) either the system is nonmagnetic and, hence, does not possess a net magnetic moment or (b) the compensation of spin densities, as in the case of antiferromagnetic material, forbids the development of polarized moment, typically observable in PNR measurements. Nd is a known antiferromagnet [17], which can explain the absence of irreversibility between the $R^{+}$and $R^{-}$curves in the zero field. However, a small separation between $R^{+}$and $R^{-}$curves is observed in the PNR measurement in applied field, Fig. 3(b), which indicates the development of a field-induced magnetic moment in the $\mathrm{Nd}$ film. The magnetization profile is uniform through the film thickness. So no interface effect is detected.

The quantitative determination of the overall magnetization in the applied field is obtained by analyzing the experimental results using a generic LICORNE-PY program 
[30,31], which generates the reflectivity pattern for a given set of physical parameters of the system, e.g., layer thickness, density, interface roughness, and magnetic moment of magnetic layer. The fitting was performed simultaneously to the data sets measured at different temperatures and fields. This procedure ensures the high accuracy of obtained parameters. Nuclear and magnetic scattering length densities (NSLD and MSLD), corresponding to the depth profiles of the chemical structure and in-plane magnetization vector distributions, respectively, are depicted in Figs. 3(d) to 3(f). The magnetization of Nd thin film is manifested by the spin asymmetry SA, given by $\left(R^{+}-R^{-}\right) /\left(R^{+}+R^{-}\right)$. The experimental plot of spin asymmetry SA as a function of wave-vector transfer $Q$ at different temperatures and fields are shown in Figs. 3(g) to 3(i). As expected, very small MSLD, corresponding to $3.4 \mathrm{emu} / \mathrm{cc}$ and $1.2 \mathrm{emu} / \mathrm{cc}$ were detected in experimental data at $T=5 \mathrm{~K}$ and $T=30 \mathrm{~K}$ in the low field of $20 \mathrm{Oe}$, respectively. On the other hand, a MSLD with the net moment of $19.6 \mathrm{emu} / \mathrm{cc}$ is extracted from the field measurement data at $T=5 \mathrm{~K}$ in Fig. 3(h). An analysis of PNR data not only confirms the notion of field-induced magnetism in the $\mathrm{Nd}$ thin film, but also reveals the absolute value of magnetic moment at $H=1.2 \mathrm{~T}$. Field application aligns the neighboring moment towards field direction. A similar effect has been previously reported in antiferromagnetic materials [32].

We also tried to understand the electrical characteristics and the absence of magnetism in zero field in the $\mathrm{Nd}$ film using the density functional theory (DFT) calculations. DFT calculations are performed for both the bulk and the thin film specimens of Nd. Although the study of bulk Nd is not the main focus of our study, it helps us in cross-checking the DFT results using the previously known parameters. DFT calculations are performed by employing the plane-wave basis set, as implemented in the QUANTUM-ESPRESSO [33]. The projector-augmented wave method was used with the Troullier-Martins norm-conserving pseudopotential with nonlinear core correction. The exchange correlation functional was treated within the generalized gradient approximation of the Perdew-Burke-Ernzerhof-generalized gradient approximation (PBE-GGA) [34]. A well-converged kinetic-energy cutoff of 60 Ry was used with a Monkhorst-Pack sampling of $16 \times 16 \times 4(16 \times 16 \times 1)$ for the bulk (thin-film) case. Previously reported $\mathrm{Nd}$ lattice parameters were used as the initial configuration of the atoms for the bulk case [35]. For thin film calculations, we used the supercell approximation and adopted the truncated Coulomb interaction method proposed in Ref. [36]. For both bulk and thin film calculations, adjacent layers of $\mathrm{Nd}$ atoms were initialized to be antiferromagnetic. A strict self-consistent energy convergence criterion of $5 \times$ $10^{-8} \mathrm{Ry}$ is imposed. Figures 4(a) and 4(c) show the calculated hole-type spin-resolved Fermi surfaces for the majority (spinup) and minority (spin-down) carriers in the bulk case. One can see the difference in Fermi pockets which branches out of the highly symmetric $\mathbf{k}$ points: $M$ and $K$. In the case of the majority spin, as shown in Fig. 4(a), the Fermi surface extends from the $M$-point towards the $\Gamma$-point, forming a centered pocket, whereas in the minority spin case, shown in Fig. 4(c), the Fermi pockets are clustered at the $K$-point and also centered at the $\Gamma$-point. On the other hand, the majority and minority Fermi surfaces in the thin film, as shown in Figs.

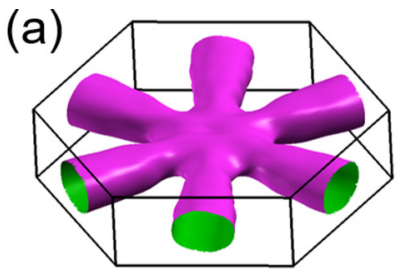

(b)

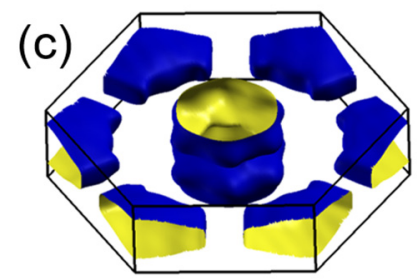

(e)
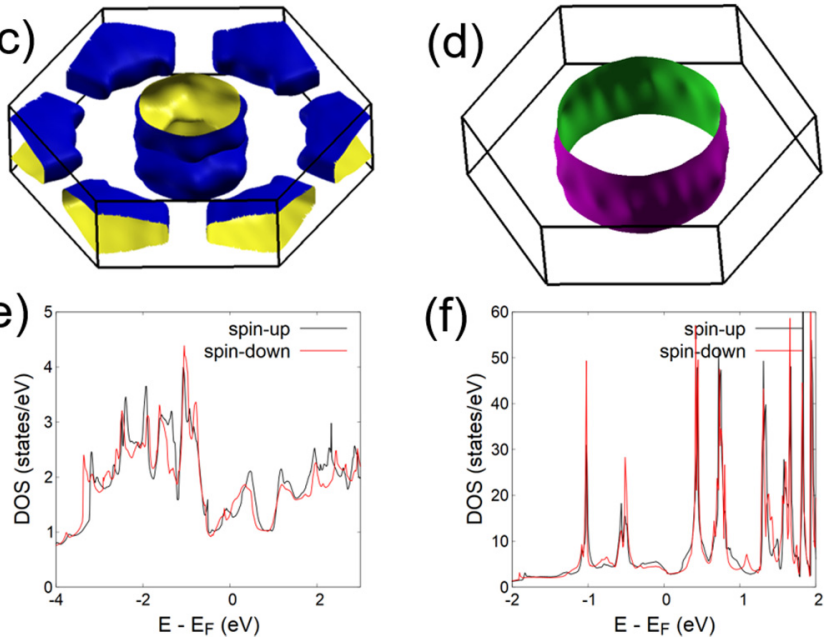

(f)

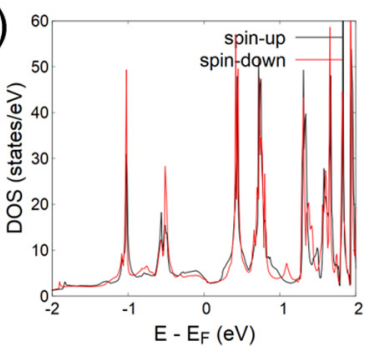

FIG. 4. Fermi surface properties of bulk and thin film specimens of Nd. (a)-(d) Spin-resolved Fermi surfaces of bulk and thin-film of Nd, respectively: (a), (b) spin up and (c), (d) spin down. (e), (f) Density of states of bulk and thin-film of $\mathrm{Nd}$, calculated using density functional theory (see text), elucidates the semiconducting character of the material. It is consistent with electrical measurements, exhibiting very weak metallic conductivity.

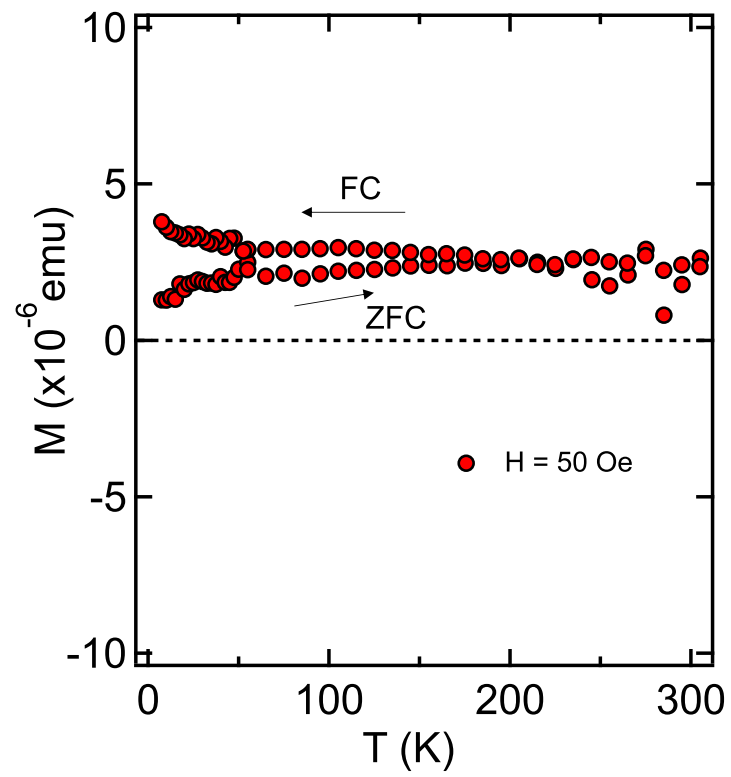

FIG. 5. Magnetization measurements of Nd thin film. Magnetization as a function of temperature is plotted at 50 Oe of applied field value. Magnetic measurement reveals very small net magnetization $\sim 2 \times 10^{-6}$ emu, Also there is very weak irreversibility between ZFC and FC (field cool) curves. Interestingly, there is a downward trend towards zero magnetization in ZFC curve at low temperature, which may arise due to the antiferromagnetic nature of thin film in low field. 

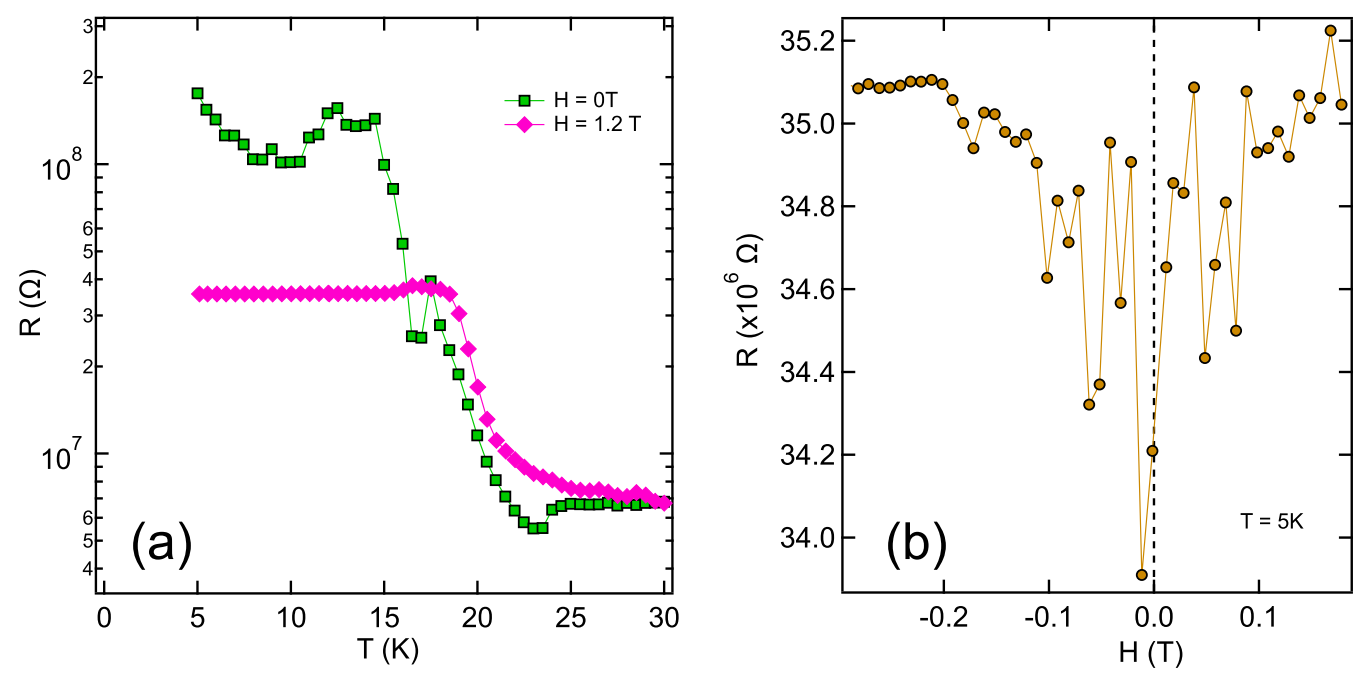

FIG. 6. (a) Electrical transport measurements of Nd thin film in zero and applied magnetic field. (b) $R$ vs. $H$ data at $T=5 \mathrm{~K}$ reproduces the fluctuations in MR at low field. The fluctuation pattern is mostly similar to that shown in Fig. 2 inset.

4(b) and 4(d), manifest a truncated cylinder-shaped pocket centered at the $\Gamma$-point, which characterizes the presence of heavy carriers. As shown in Figs. 4(e) and 4(f), the calculated spin-resolved density of states (DOS) in bulk Nd exhibits a weak splitting between the majority and minority states, whereas there is a close overlap in the case of the thin film. This indicates that the bulk Nd is antiferromagnetic in nature. The difference in electrical conductivity of the bulk $\mathrm{Nd}$ and the thin film Nd is manifested in the DOS at the Fermi level. In thin film Nd, the DOS at the Fermi level resembles the delta function and is much higher than that of the bulk Nd. It suggests the existence of a much larger carrier effective mass in the thin film case, which gives rise to much weaker electrical conductivity. This is in qualitative agreement with experimentally observed low electrical conductivity in $\mathrm{Nd}$ thin film.

Rare earth elements are of strong technological importance. However, a strong understanding of physical and magnetic properties in both the bulk and thin film specimens of an element is necessary for successful practical implications. The reconfiguration of the Fermi surface in the thin film may cause drastically different properties, compared to the bulk material. An example to this fundamental effect is demonstrated in this paper. In summary, we presented a comprehensive study of Nd thin film using electrical, magnetoresistance, and polarized neutron reflectometry measurements. Our study elucidates important information regarding the electric and magnetic properties in $\mathrm{Nd}$ thin film that can play a crucial role in the practical applications. Unlike bulk Nd, the thin film of $\mathrm{Nd}$ is not very electrically conducive. At low temperature of $18 \mathrm{~K}<T<25 \mathrm{~K}$, the resistivity registers a sharp increase due to the hopping of charge carriers between different sites. But it is not an insulator. This is a desirable property for many applications, such as in developing the high performance electrocatalyst. We also showed that, as grown, Nd film does not possess net magnetization. In the applied field, a weak magnetic moment, indicating weak ferromagnetism, develops at low temperature $T<18 \mathrm{~K}$. The remnant characteristic of field-induced magnetization causes the occurrence of competing quasistable states near zero field when magnetic field is reduced to $H=0 \mathrm{~T}$ from a higher value. The experimental observation of the absence of magnetic moment in the zero field is also confirmed by the DFT calculations. Future works on the study of the Nd film grown on a different substrate, such as $\mathrm{SrTiO}_{3}$, are highly desirable. Perhaps, a highly anisotropic substrate can cause an experimentally observable polarization of net magnetic moment in zero field.

D.K.S. thankfully acknowledges the support by the Department of Energy, Office of Science, Office of Basic Energy Sciences under Grant No. DE-SC0014461. The research conducted at the Spallation Neutron Source was sponsored by the Scientific User Facilities Division, Office of Basic Energy Sciences, U.S. Department of Energy.

\section{APPENDIX}

In Fig. 5, we plot the magnetization data of the neodymium thin film. Magnetization data are obtained using conventional zero-field cool (ZFC) and field-cool (FC) procedure in low magnetic field application of $H=50$ Oe. A small magnetic field ensures that the local moment character is preserved. Figure 6 depicts the reproducibility of electrical properties in the $\mathrm{Nd}$ thin film. Both the $R$ versus $T$ data in the zero and applied field and $R$ versus $H$ measurement near zero field reproduces the important features of previously reported experimental observations in the main text, shown in Figs. 1 and 2. 
[1] V. Zepf, Rare Earth Elements, A New Approach to the Nexus of Supply, Demand and Use: Exemplified Along the uUse of Neodymium in Permanent Magnet (Springer-Verlag, Berlin, 2013).

[2] J. Herbst and J. Croat, J. Mag. Mat. Mag. 100, 57 (1991).

[3] K. Murase, S. Hiratsuka, R. Song, and Y. Takeuchi, Jap. J. Appl. Phys. 53, 067001 (2014).

[4] Y. Kawamura, Y. Hasegawa, M. Iwamuro, T. Kitamura, and S. Yanagida, Appl. Phys. Lett. 74, 3245 (1999).

[5] S. Chinn, H. Hong, and J. Pierce, Laser Focus 12, 64 (1976).

[6] B. Sprecher, R. Kleijn, and G. Kramer, Environ. Sci. Technol. 48, 9506 (2014).

[7] Z. Lin et al., Appl. Phys. Lett. 107, 112108 (2015).

[8] A. Kemp, G. Valentine, and D. Burns, Prog. Quantum Electron. 28, 305 (2004).

[9] R. Taylor, T. McGuire, J. M. D. Coey, and A. Gangulee, J. Appl. Phys. 49, 2885 (1978).

[10] G. Sala, M. B. Stone, B. K. Rai, A. F. May, C. R. Dela Cruz, H. Suriya Arachchige, G. Ehlers, V. R. Fanelli, V. O. Garlea, M. D. Lumsden, D. Mandrus, and A. D. Christianson, Phys. Rev. Mater. 2, 114407 (2018).

[11] J. Xu, A. T. M. N. Islam, I. N. Glavatskyy, M. Reehuis, JensUwe Hoffmann, and B. Lake, Phys. Rev. B 98, 060408(R) (2018).

[12] Y. Zhang, J. Wang, F. Nan, and Q. Wang, RSC Adv. 8, 20056 (2018).

[13] Y. H. Wang, Appl. Phys. Lett. 84, 1686 (2004).

[14] M. C. Hatnean, M. R. Lees, O. A. Petrenko, D. S. Keeble, G. Balakrishnan, M. J. Gutmann, V. V. Klekovkina, and B. Z. Malkin, Phys. Rev. B 91, 174416 (2015).

[15] O. Benton, Phys. Rev. B 94, 104430 (2016).

[16] Y. Chen, B. Summers, A. Dahal, V. Lauter, G. Vignale, and D. K. Singh, Adv. Mater. 31, 1808298 (2019).

[17] P. Bak and B. Lebech, Phys. Rev. Lett. 40, 800 (1978).

[18] R. Moon, J. Cable, and W. Koehler, J. Appl. Phys. 35, 1041 (1964).

[19] J. Alstad, R. Colvin, S. Legvold, and F. Spedding, Phys. Rev. 121, 1637 (1961).
[20] N. James, S. Legvold, and F. Spedding, Phys. Rev. 88, 1092 (1952).

[21] V. Lauter, H. Ambaye, R. Goyette, W. Lee, and A. Parizzi, Phys. B: Condens. Matter 404, 2543 (2009).

[22] Z. Zeng, M. Greenblatt, M. A. Subramanian, and M. Croft, Phys. Rev. Lett. 82, 3164 (1999).

[23] L. Berger, J. App. Phys. 49, 2156 (1978).

[24] D. Parker, M. Green, S. Bramwell, A. Wills, J. Gardner, and D. Neumann, J. Am. Chem. Soc. 126, 2710 (2004).

[25] J. Gunasekera, Y. Chen, J. W. Kremenak, P. F. Miceli, and D. K. Singh, J. Phys.: Cond. Matt. 27, 052201 (2015).

[26] J. Gunasekera, Y. Chen, A. Dahal, J. Rodriguez, L. Harriger, S. Thomas, T. Heitmann, V. Dugaev, A. Ernst, and D. K. Singh, Adv. Sci. 5, 1700978 (2018).

[27] H. Negishi, H. Yamada, K. Yuri, M. Sasaki, and M. Inoue, Phys. Rev. B 56, 11144 (1997).

[28] P. M. Levy and S. Zhang, Phys. Rev. Lett. 79, 5110 (1997).

[29] V. Lauter, H. Lauter, A. Glavic, B. Toperverg, Reference Module in Materials Science and Materials Engineering (Elsevier, Amsterdam, 2016), pp. 1-27.

[30] G. Yumnam, Y. Chen, J. Guo, J. Keum, V. Lauter, and D. K. Singh (private communication)

[31] V. Lauter, A. Savici, S. Hahn, Licorne-Py: A polarized neutron specular reflectivity fitting software (2019), https://github.com/ neutrons/Licorne-Py.

[32] X. Marti, I. Fina, C. Frontera, J. Liu, P. Wadley, Q. He, R. J. Paull, J. D. Clarkson, J. Kudrnovský, I. Turek, J. Kunë, D. Yi, J.-H. Chu, C. T. Nelson, L. You, E. Arenholz, S. Salahuddin, J. Fontcuberta, T. Jungwirth, and R. Ramesh, Nat. Mater. 13, 367 (2014).

[33] P. Giannozzi et al., J. Phys.: Cond. Matt. 21, 395502 (2009).

[34] J. P. Perdew, K. Burke, and M. Ernzerhof, Phys. Rev. Lett. 77, 3865 (1996).

[35] F. H. Spedding, J. J. Hanak, and A. H. Daane, J. Less Common. Met. 3, 110 (1961).

[36] T. Sohier, M. Calandra, and F. Mauri, Phys. Rev. B 96, 075448 (2017). 